\title{
Improving topology optimization intuition through games
}

Nobel-Jørgensen, Morten; Malmgren-Hansen, David; Bærentzen, J. Andreas; Sigmund, Ole; Aage, Niels

Published in:

Structural and Multidisciplinary Optimization

Link to article, DOI:

10.1007/s00158-016-1443-0

Publication date:

2016

Document Version

Peer reviewed version

Link back to DTU Orbit

Citation $(A P A)$ :

Nobel-Jørgensen, M., Malmgren-Hansen, D., Bærentzen, J. A., Sigmund, O., \& Aage, N. (2016). Improving topology optimization intuition through games. Structural and Multidisciplinary Optimization, 54(4), 775-781. https://doi.org/10.1007/s00158-016-1443-0

\section{General rights}

Copyright and moral rights for the publications made accessible in the public portal are retained by the authors and/or other copyright owners and it is a condition of accessing publications that users recognise and abide by the legal requirements associated with these rights.

- Users may download and print one copy of any publication from the public portal for the purpose of private study or research.

- You may not further distribute the material or use it for any profit-making activity or commercial gain

- You may freely distribute the URL identifying the publication in the public portal 


\title{
Improving topology optimization intuition through games
}

\author{
Morten Nobel-Jørgensen - David Malmgren-Hansen · J. Andreas \\ Bærentzen · Ole Sigmund · Niels Aage
}

Received: date / Accepted: date

\begin{abstract}
This paper describes the educational game, TopOpt Game, which invites the player to solve various optimization challenges. The main purpose of gamifying topology optimization is to create a supplemental educational tool which can be used to introduce concepts of topology optimization to newcomers as well as to train human intuition of topology optimization. The players are challenged to solve the standard minimum compliance problem in 2D by distributing material in a design domain given a number of loads and supports with a material constraint. A statistical analysis of the gameplay data shows that players achieve higher scores the more they play the game. The game is freely available for the iOS platform at Apple's App Store and at http://www . topopt.dtu.dk/?q=node/909 for Windows and OSX.
\end{abstract}

Keywords Interactive - Topology optimization - Gamification - Smartphones - Tablets · PDE constrained optimization

\section{Introduction}

Topology optimization has for the past two and a half decade made a great impact on the design of struc-

The authors acknowledge the support from the Villum foundation through the NextTop project.

M. Nobel-Jørgensen · D. Malmgren-Hansen · J. A. Bærentzen Department of Applied Mathematics and Computer Science, Technical University of Denmark, Asmussens Alle,

B.305, 2800 Kgs. Lyngby, Denmark

E-mail: mono@dtu.dk

N. Aage $\cdot$ O. Sigmund

Department of Mechanical Engineering, Solid Mechanics,

Technical University of Denmark, Nils Koppels Alle,

B.404, 2800 Kgs. Lyngby, Denmark tures and mechanical elements. The method is used in many different fields of engineering and can be applied on many different scales, from designing microstructures to large-scale constructions such as ships, skyscrapers and aircrafts. Topology optimization is usually performed as a discrete step in the design process - often integrated with CAD software. Even though topology optimization algorithms are able to find good solutions to most problems, it is important for their users to have a good intuition for the method in order to get a feel of the process and be able to identify cases where the algorithms clearly get stuck at a local minimum. In cases where topology optimization is not used in the design process, due to time or resource constraints, the final design relies on performance of the "human topology optimization", where a good intuition is critical.

This article describes how we have transformed the topology optimization problem into a game in order to train human intuition for the problem. The game can be used as an educational game in topology optimization lectures - or it can be played by people with no experience in the field. By anonymously tracking players' progress in the game we are able to estimate people's topology optimization intuition and how this intuition progresses the more times a single user plays.

By gamifying topology optimization we are also aiming at heightening the awareness of the field for a broader audience.

\section{Related work}

Gamification, where game elements and game design are added to non-game contexts, can be used to improve the user experience and user engagement [4]. Gamifica- 
tion is used in various places, including science, where it has helped research in protein folding. Computing how proteins fold is a hard non-convex optimization problem, and finding good foldings is crucial for understanding, and potentially curing, diseases like Alzheimer's, Parkinson's and some types of cancers. Two famous gamification projects using protein folding are:

- Folding@home turns consumers' computers into one big distributed computer by utilizing the computer's idle time to perform the heavy computations. Folding@home uses a scoreboard (both personal and team-based) as a way to motivate people to run the software on their computer [2].

- FoldIt has a different take on protein folding. The creators have turned protein folding into a game by abstracting the mathematical problem into easily understandable metaphors. In FoldIt, users compete (individually or in groups) to come up with the best folding. In some cases, the player performance can even beat the solutions found by existing protein algorithms. User behavior in FoldIt has been studied in order to extract strategies to improve the protein folding algorithms [5].

The two protein-folding projects also have the positive side effect of increasing people's awareness of the protein-folding problem and its related diseases.

The work presented here is related to the TopOpt app [1], where topology optimization is solved in an interactive manner. Some elements of the user interface have been reused as well as parts of the topology optimization kernel. One feature that has not been reused is the multiresolution topology optimization scheme MTOP [6], since this method does not penalize solutions which are not watertight. This had the implication that solutions where the material was separated by an empty row or column on the fine grid were evaluated as if they were connected.

The TopOpt Game app was launched for iOS and PC on the 28th of August 2014.

\section{Problem formulation}

The optimization problem we ask the player to solve in the TopOpt Game is the standard minimum compliance design problem for linear elasticity [3]. Following a finite element (FE) discretization, the classical topology optimization problem can be stated in a discrete form using the density approach [8] as

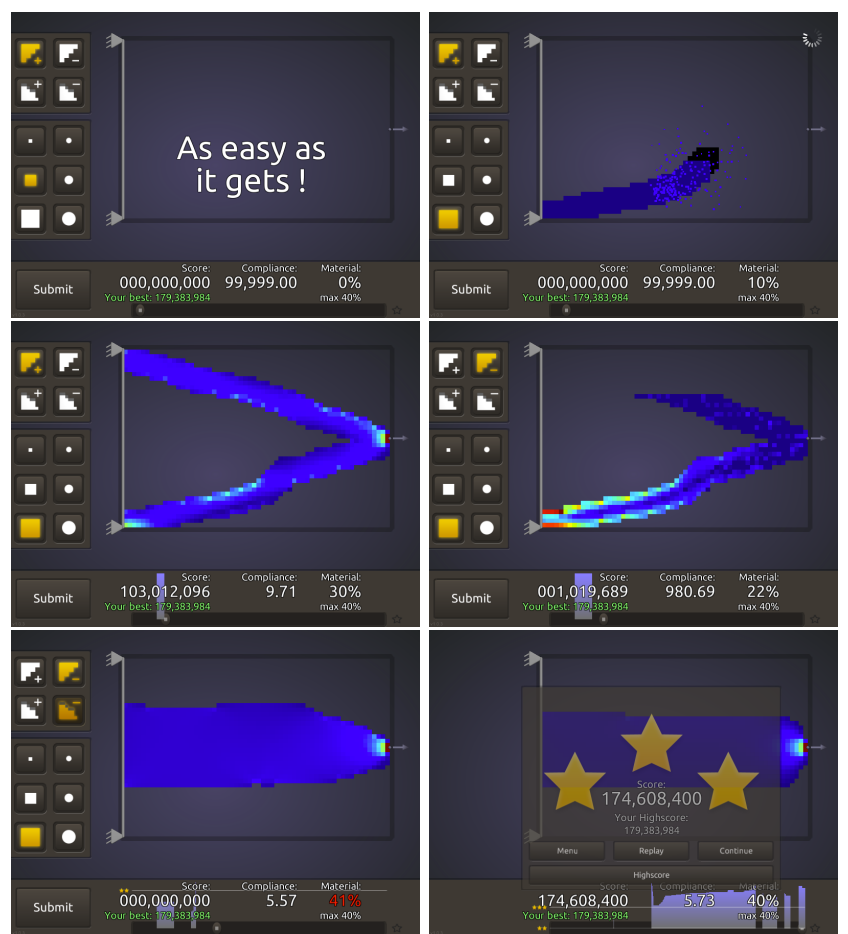

Figure 1: Gameplay: While the user paints a solution the game provides feedback in terms of a score and by visualizing the strain energy density using the jet color scheme.

$$
\begin{array}{ll}
\min _{\rho \in \mathbb{R}^{n}} & \phi(\boldsymbol{u}, \boldsymbol{\rho})=\boldsymbol{F}^{\boldsymbol{T}} \boldsymbol{u} \\
\text { s.t. } & \boldsymbol{K}(\boldsymbol{\rho}) \boldsymbol{u}=\boldsymbol{F} \\
& V(\boldsymbol{\rho}) / V^{*}-1 \leq 0 \\
& \rho_{i} \in\left\{\rho^{\min }, 1\right\}, i=1, \ldots, n
\end{array}
$$

where $\phi=\boldsymbol{F}^{\boldsymbol{T}} \boldsymbol{u}$ is compliance, $\boldsymbol{\rho}$ is a vector of $n$ element densities (design variables), $\boldsymbol{u}$ and $\boldsymbol{F}$ are the nodal displacement and force vectors, respectively, $\boldsymbol{K}(\boldsymbol{\rho})$ is the global stiffness matrix, and $V(\boldsymbol{\rho}) / V^{*}-1 \leq 0$ is the volume constraint. Finally, $\rho^{\text {min }}$ is a lower bound on the design variables.

During level design, a baseline compliance is found using the solver from [1], where each design variable can have a value between $\rho^{\min }$ and 1 . The baseline compliance is used for rating the player performance in terms of $0-3$ stars. The actual rating mapping is adjusted to the difficulty of each problem during level design.

\section{Game design and implementation}

TopOpt Game is inspired by puzzle-games (a genre of computer games), which constantly challenge the players and give rewards when progress is made. This en- 


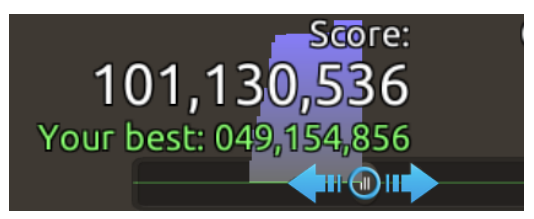

Figure 2: Score graph: Sliding the handle left or right allows easy backtracking.

gagement loop will take the player on a journey starting with simple problems with few supports and a single load and gradually increases the difficulty by adding more loads, restrictions on the design domain, distributed loads and multiple load cases.

The goal of the game is to distribute a constrained amount of material in a design domain in order to minimize compliance (and hence maximize stiffness). The player must find the best material distribution which connects all the loads with the relevant support regions before a timer runs out. Figure 1 shows a typical gameplay. If too much material has been used, the player is penalized by setting the score to zero.

The way material is distributed is inspired by brush strokes in painting programs; The player selects the add or remove material state and a brush type, and then makes a drag-gesture in the design domain. There also exist two specialized tools which add or remove elements only on the boundary of the structure. As a visual cue, a particle effect helps illustrate how elements are constructed or dissolved when using the draw tools. Note that the design elements within the design domain either have material or are void; "graylevel" elements are not allowed in order to simplify the user interaction.

To ensure a good responsiveness of the user interface, we use multiple threads. The main thread is responsible for updating the user interface, listening for events, and rendering the game. Another thread evaluates the compliance of the current structure in an asynchronously way. When the compliance has been evaluated, the value is displayed to the player as well as a score (a scaled multiplicative inverse of the compliance). We found that maximizing a value (score) is a much more intuitive goal than minimizing a value.

The score is the most important user interface element and it is very important that the player is able to see if a change has a positive or negative consequence. For this reason the score is visualized in two complimentary ways:

- A score label which shows the score to the player as an integer number with a dot delimiter for easy reading. This makes it straightforward to compare the current score with the highscore (also shown as a label).
- A score graph which allows the player to see the development of the score over time, as shown in 2. Changes to the graph are smoothly animated, which makes it easy to grasp when the score increases. The score graph also shows three important score milestones as horizontal lines for a two-star rating, a three-star rating and previous highscore (if any).

Besides visualizing the score, the score graph also serves two other purposes: It works as the game timer, showing the player how much time remains, and the handle below the graph allows the player to easily backtrack the solution to any previous evaluated state by dragging the handle to a previous time point.

When the player is out of time, or is submitting the level, the score is compared to a baseline score and the player's solution is rated from 0 to 3 stars. We use this simplified rating system to give a clear indication of the player performance.

The player is also able to compete against himself or herself, trying to beat his/hers best score. In addition, the game is on iOS integrated with GameCenter. Using GameCenter, a detailed leaderboard for each level is available as well as the option of challenging friends if a good solution to a level is found.

When a level has been played, gameplay information is sent to a server. The gameplay information contains all information about the player performance during gameplay, including every evaluated action that the player performs as well as score and compliance of each action.

\section{Level editor}

One of the biggest challenges in creating the game was how to create interesting and fun topology optimization problems. One option would be to create procedurally generated levels containing topology optimization problems created by parameters such as difficulty and time complexity. However, we found that this idea would be a too hard problem to solve and instead decided to manually craft the levels.

To make level design easy, we have included a level editor similar to the user-interface of the TopOpt app [1]. The level editor has been used to design the predefined levels but can also be used by the players to experiment with new challenges.

A user can create a level by defining a rectangular size of the design domain followed by removing or adding elements to allow non-rectangular design domains.

The level is then augmented with loads and supports, which are inserted onto the nodes of the grid and 


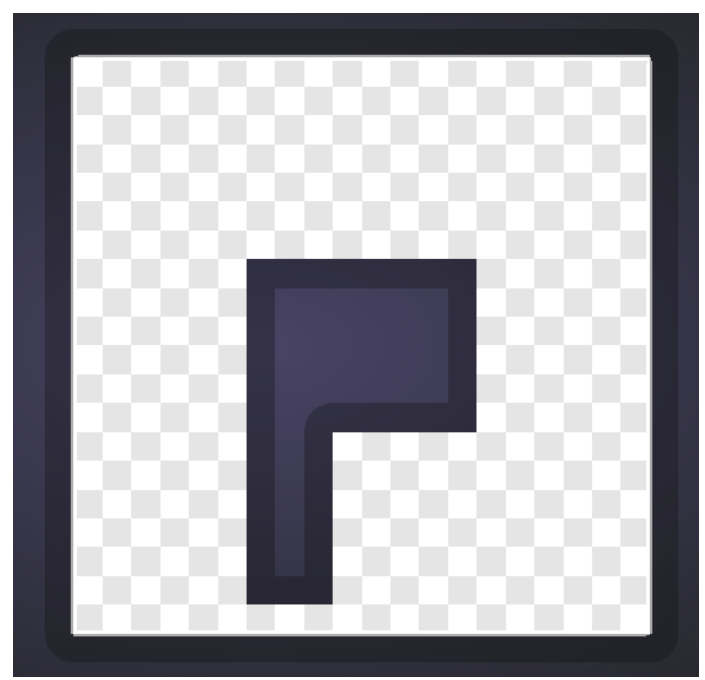

(a) Defining the design domain (size and excluded elements). The checkerboard shows the actual element size.

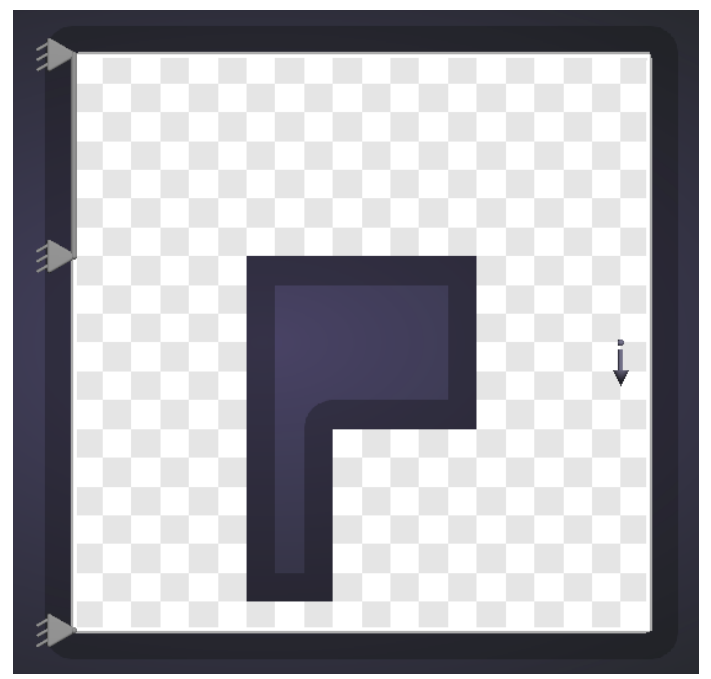

(b) Adding loads and supports

Figure 3: Besides solving predefined levels, the players can also design new levels using the built-in level editor. Apart from the design domain and location of loads and supports shown here, additional information, such as volume constraint and time, need to be defined.

optionally distributed horizontally or vertically. Figure 3 shows the steps of designing a level.

When a level has been designed and some additional properties (time, name, category, volume fraction) have been specified it can be play-tested to find out if it works as intended.

User-generated levels are private and only visible to the player who created them. However, the user has the option to suggest a custom level as a global level, and in time this will increase the number of global levels.
Members of the DTU TopOpt group created the initial global levels in the game. The levels include classic topology optimization problems such as the cantilever and L-shaped cantilever. The levels have been designed such that they are all unique and do not exist in mirrored or rotated versions. A recent addition to the set of levels is the Zhou-Rozvany problem [9], which is interesting since it is one of the few cases where we know the global optimum and where many numerical TopOpt approaches fail.

The global levels are sorted by their estimated difficulty, such that easy levels are first in the list presented to the player. The level difficulty is estimated by the level designer based on the design domain, loads and support as well as playtesting of the level during design. The level designer is also responsible for adjusting which score gives a one, two or three-star rating. The actual level difficulty is hard to quantify due to the complexity of topology optimization problems and due to differences in player skills and experiences.

\section{Results}

\subsection{Data analysis}

To evaluate whether playing the game actually improves players' intuition about topology optimization, we have analyzed the gameplay data to find a relationship between the number of games a person has played and the score he or she achieves. Since the analysis is performed on data from uncontrolled usage, we make the following assumptions:

1. Each player (registered or unregistered) corresponds to a single person.

2. In each completed gameplay the player strives for the maximum score.

3. There is a correlation between obtaining a high score in the game and having a good intuition about topology optimization. Hereby we neglect the performance gain of both learning the user interface, as well as known solutions from previous gameplays of the same or similar kind.

The following analysis is based on gameplay data from the global levels. It consists of gameplay observations with the variables listed below:

- Player ID

- Level ID

- Experience (number of games the player has played prior to current gameplay).

- Score (The score is the reciprocal of compliance. We have chosen to normalize this with the maximum 
score achieved at the current Level ID for the statistical analysis).

A gameplay sometimes has the final score of 0 . This usually means that the player broke the volume constraint at end of the game and did not have time to undo the action. We found that these cases do not tell us much about the player's topology optimization intuition and therefore removed such observations from the analysis. However, the analysis does contain cases where the score is close to 0 , which occurs when a load is not fully supported.

In a controlled experiment where all players had played an equal amount of gameplays the analysis of score improvement would be easier. In our experiment players play as long as they want to and though presented with a fixed list of levels, they also decide the order of and which levels they play individually. Due to this we set up some conditions for observations that we include in our analysis. We only include observations from levels that have been played more than 50 times. Furthermore, we disregard observations from players that in total have 5 or less gameplays. At the time of writing only 11 out of 708 registered players have more than 100 gameplays. This leaves Experience levels above 100 with higher uncertainty and we therefore also exclude these observations from our analysis.

Out of 12467 collected gameplays this leaves us with 6962 observations where almost 4000 gameplays were removed by the 0 score constraint.

Several approaches to show the relationship between score and experience can be taken, but if fitting a linear model to the observations it is important to take into account that players cannot be assumed to have equal skills prior to playing this game. Another fact is that individual levels have different difficulty and this might affect the rate at which score increases.

A Linear Mixed-Effect (LME) model is one way of coping with the fact that some variables might be sources of random variation. Figure 4 a shows a plot of all observations (gameplays) across levels and of all players that fulfil our conditions. A LME model fit is also shown in the figure with a slope of 0.0024 . Alternatively, we could look at observations from a single level alone and leave out the variation introduced by level difficulty. This is shown in Figure $4 \mathrm{~b}$ for the level with the most gameplays. The LME model gives a higher slope fitted to these data, but the reason for this level having the most observations is likely to be that it is one of the first on the list of levels a player is introduced to and thereby also one of the easier levels.

Even though we achieve significant parameters $(<0.001)$ in the model for both slope and intercept, it is visually clear that the data is somewhat noisy. This is due to the

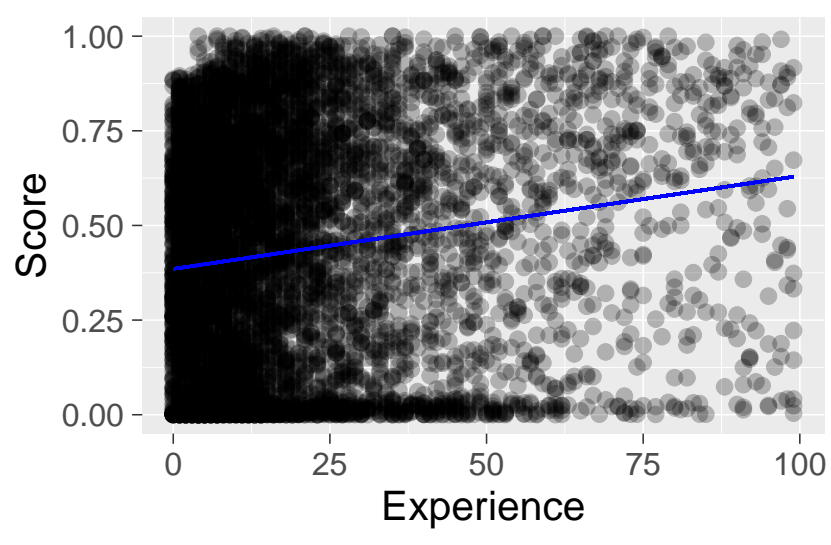

(a) 6962 observations plotted. Linear Mixed-Effect model fit (Blue line).

slope $=0.0024\left(p_{\text {value }}<0.001\right)$

intercept $=0.39\left(p_{\text {value }}<0.001\right)$

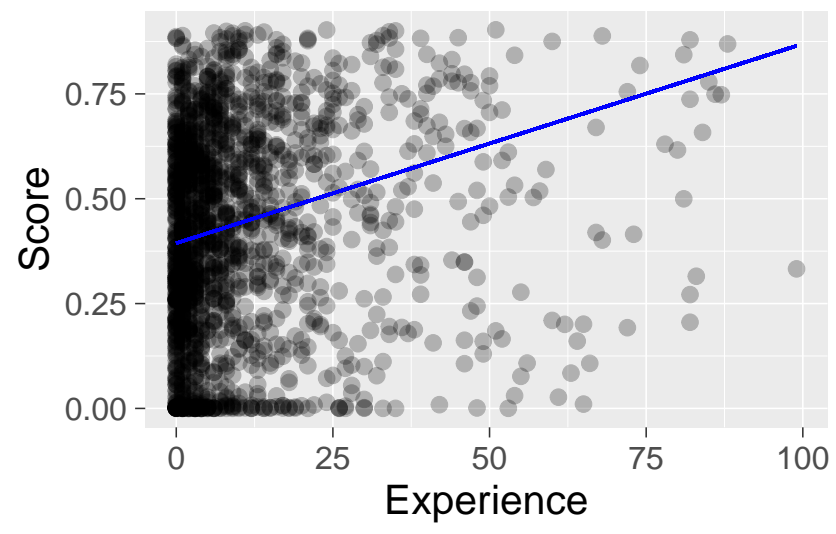

(b) 1708 observations plotted from the Level with most gameplays. Linear Mixed-Effect model fit (Blue line).

slope $=0.0048\left(p_{\text {value }}<0.001\right)$

intercept $=0.39\left(p_{\text {value }}<0.001\right)$

Figure 4: Plots of Score relative to Experience. Figure a: observations across all levels. Figure b: observations from the single level with most gameplays.

fact that, even though players averagely improve with experience, they tend to still have occasionally "bad" gameplays with low score (see plots on Figure 4).

It could therefore be relevant to look at the absolute mean curve of Score versus Experience. In Figure 5 these numbers are plotted along with a linear regression fit.

Table 1 shows the values plotted in Figure 5 along with the variance and number of observations for each interval of Experience.

The fact that the data is so unevenly distributed across experiences (seen from the "Observations" col) umn in Table 1) together with the fact that 1984 out 6962 observations have been played on a single level out of the 20 levels included in this analysis leads to 


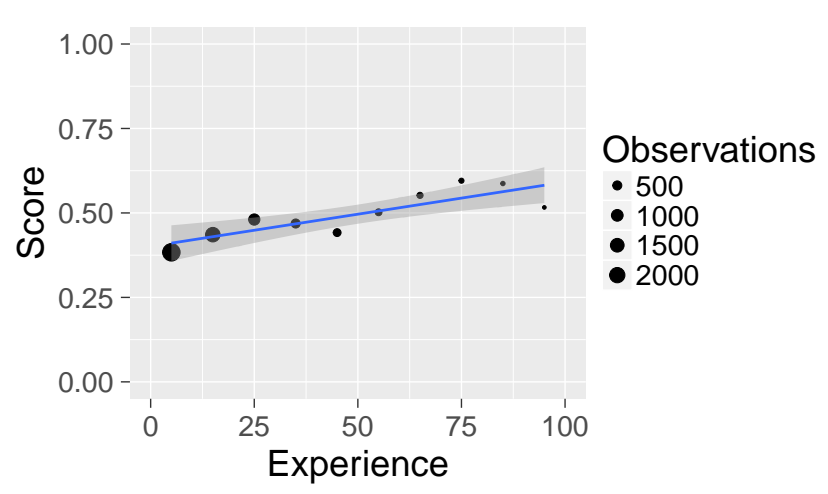

Figure 5: Linear Regression on averaged data with 95\% confidence intervals. The data points on this plot can be seen in Table 1.

slope $=0.0024\left(p_{\text {value }}=0.001\right)$

intercept $=0.40\left(p_{\text {value }}<0.001\right)$

$R^{2}=0.60$

\begin{tabular}{|r|r|r|r|}
\hline Experience & Mean Score & Variance & Observations \\
\hline $0-10$ & 0.38 & 0.08 & 2802 \\
\hline $10-20$ & 0.44 & 0.10 & 1747 \\
\hline $20-30$ & 0.48 & 0.10 & 898 \\
\hline $30-40$ & 0.47 & 0.10 & 493 \\
\hline $40-50$ & 0.45 & 0.11 & 333 \\
\hline $50-60$ & 0.50 & 0.11 & 229 \\
\hline $60-70$ & 0.55 & 0.10 & 171 \\
\hline $70-80$ & 0.60 & 0.10 & 113 \\
\hline $80-90$ & 0.59 & 0.09 & 92 \\
\hline $90-100$ & 0.52 & 0.11 & 84 \\
\hline
\end{tabular}

Table 1: Normalized score averaged across all players and all levels. Observations are from 611 players across 20 different levels.

some uncertainty. From this data we do not believe we can tell the exact learning rate of solving the topology optimization tasks presented, but we can conclude that there is a positive correlation between how many games one play and the score that is achieved.

\subsection{Classroom evaluation}

The game has been used in the undergraduate course "Mechanics and Materials" at the Technical University of Denmark in order to evaluate its use as a learning aid. The learning objectives of using the game was for the students to be able to understand the basic concepts of topology optimization. The game was introduced shortly after the students' first acquaintance with topology optimization. After a short 5-minutes introduction to the user interface, the students played the game for 30 minutes as seen in Figure 6 .
During the 30 minutes of gameplay some of the students tried to complete as many levels as possible whereas others strove to get a three-star ranking for each level.

Afterwards the students were asked to complete a short anonymous questionnaire which 46 of the (approximately) 50 attending students answered. Most importantly the questionnaire revealed that the students in general found that the game was a good supplemental teaching aid (Table 2) and that it has an appropriate difficulty (Table 3).

Figure 7 shows the data gathered in the classroom evaluation. The result is in line with the overall results shown in section 6.1 .

\begin{tabular}{|l|r|}
\hline Strongly disagree & $2.17 \%$ \\
\hline Disagree & $2.17 \%$ \\
\hline Neither agree nor disagree & $19.57 \%$ \\
\hline Agree & $60.87 \%$ \\
\hline Strongly agree & $15.22 \%$ \\
\hline
\end{tabular}

Table 2: Survey question: "I find that TopOpt Game works well as a supplemental teaching aid when learning about topology optimization"

\begin{tabular}{|l|r|}
\hline Way too easy & $2.17 \%$ \\
\hline Easy & $0.00 \%$ \\
\hline Have an appropriate difficulty & $50.00 \%$ \\
\hline Hard & $47.83 \%$ \\
\hline Way too hard & $0.00 \%$ \\
\hline
\end{tabular}

Table 3: Survey question: "Progression: I find that the increasing challenges from the first to the later levels is"

\section{Conclusion}

In this paper, we have presented the TopOpt Game, an educational game which allows players to learn topology optimization by finding good solutions to given problems. The game shows a new way of teaching topology optimization, by which the students get familiar with the overall concepts. Using the game as a supplement to traditional MATLAB-based teaching allows students to compete against each other and to get a feel for how hard a problem topology optimization is.

We have shown that players averagely increase their score as they become more experienced. We encountered difficulties analyzing high experience due to lack 


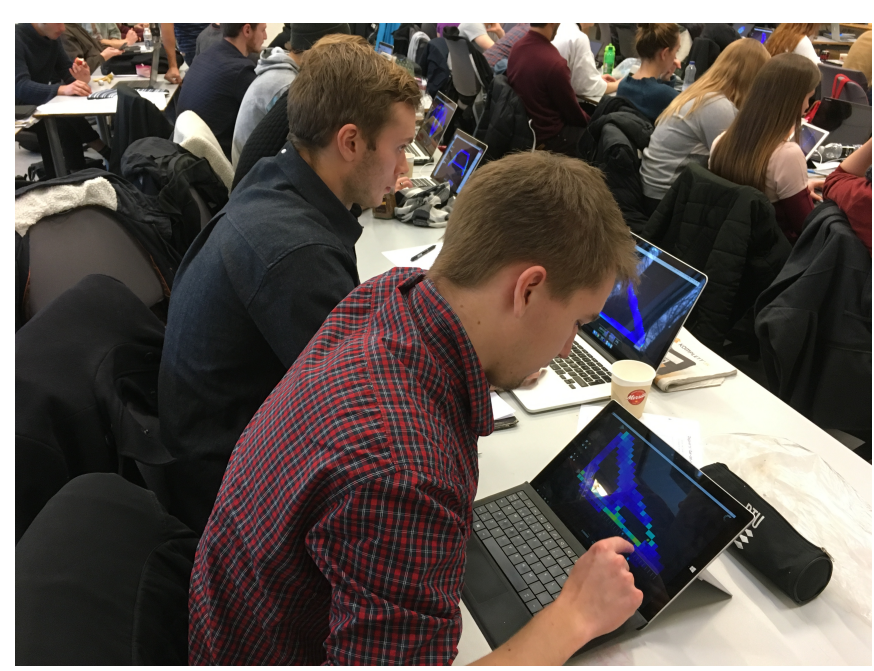

Figure 6: Classroom evaluation

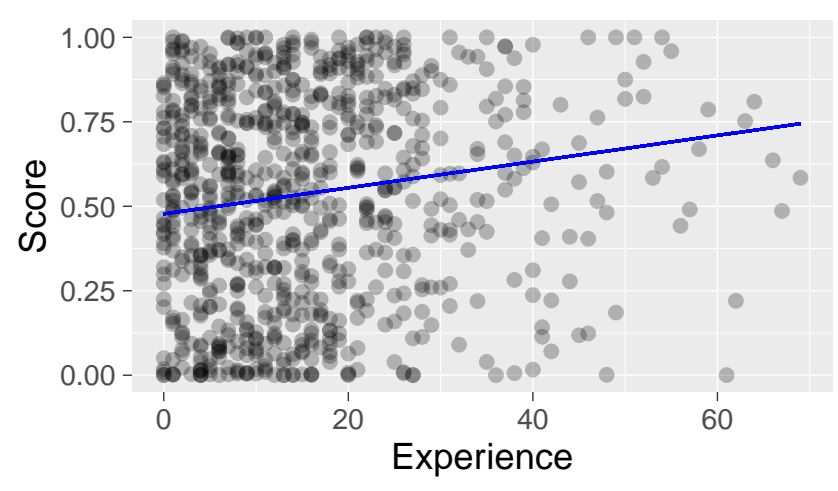

Figure 7: 820 gameplays from the classroom evaluation. Data is collected from 48 players across 17 different levels. Blue line is Linear Mixed Effect model fit with players and levels as random effects.

slope $=0.0037\left(p_{\text {value }}<0.001\right)$

intercept $=0.49\left(p_{\text {value }}<0.001\right)$

of observations here. The trend of increasing score with increasing experience seems clear though.

Other than lack of observations our problems in the analysis might be found in the assumptions we state. Referring to the Results section, assumptions 1 and 2 are critical, but probably not always met. We have no guarantee that a player corresponds to a single person and this leads to another source of uncertainty in our analysis. Also, that players may not always perform, or strive, their best in every single gameplay can mislead the analysis. Players might be distracted during playing or try a silly solution out of curiosity.

We have also shown that students find that the game is a good supplemental learning aid for topology optimization with an appropriate difficulty.
One future simplification of the game is to let the game enforce the volume constraint. This should make the gameplay slightly easier, since players have one less thing to think about and it should have a positive effect on the learning rate when the data is analyzed.

When more gameplay data has been gathered, it could be interesting to investigate the data more thoroughly to unveil the underlying strategies humans use to solve topology optimization. Potentially, this could lead to improvements of existing topology optimization algorithms. It would also be interesting to analyze the data to see if some types of problems are particular hard to solve for humans in order to identify typical pitfalls to be aware of. This includes a further investigation of the Zhou-Rozvany problem where the global optimum is known.

In relation to this, a new experiment could involve a test group of 10-20 persons. Letting these players play the game in a controlled environment would improve the chances of the first two assumptions in section 6 to be true. By testing statistically whether the test groups' performance deviates significantly from the other data, it could help reveal whether the assumptions are right. Furthermore, the group could get a set of different topology optimization tasks before and after playing the game in order to see whether it improved their skills in this.

The topology optimization game could also be extended to 3D (similar to TopOpt App 3D [7]), where voxels could be added or removed by using a painting gesture on existing material similar to the popular game, Minecraft. Moving to 3D does add some additional challenges, such as visualizing strain energy density inside a volume.

Acknowledgements The authors would like to extend their gratitude to the members of the TopOpt and NextTop groups at DTU for their invaluable input to the design and testing of the TopOpt Game.

\section{References}

1. Aage, N., Nobel-Jørgensen, M., Andreasen, C., Sigmund, O.: Interactive topology optimization on hand-held devices. Structural and Multidisciplinary Optimization 47(1), 1-6 (2013)

2. Beberg, A., Ensign, D., Jayachandran, G., Khaliq, S., Pande, V.: Folding@home: Lessons from eight years of volunteer distributed computing. In: Parallel Distributed Processing, 2009. IPDPS 2009. IEEE International Symposium on, pp. 1-8 (2009). DOI 10.1109/IPDPS.2009. 5160922

3. Bendsøe, M., Sigmund, O.: Topology Optimization: Theory, Methods and Applications. Springer (2003)

4. Deterding, S., Sicart, M., Nacke, L., O'Hara, K., Dixon, D.: Gamification. using game-design elements in non- 
gaming contexts. In: CHI '11 Extended Abstracts on Human Factors in Computing Systems, CHI EA '11, pp. 2425-2428. ACM, New York, NY, USA (2011). DOI $10.1145 / 1979742.1979575$

5. Khatib, F., Cooper, S., Tyka, M.D., Xu, K., Makedon, I., Popović, Z., Baker, D., Players, F.: Algorithm discovery by protein folding game players. Proceedings of the National Academy of Sciences 108(47), 18,949-18,953 (2011). DOI 10.1073/pnas. 1115898108

6. Nguyen, T., Paulino, G., Song, J., Le, C.: A computational paradigm for multiresolution topology optimization (mtop). Structural and Multidisciplinary Optimization 41(4), 525-539 (2010)

7. Nobel-Jørgensen, M., Aage, N., Nyman Christiansen, A., Igarashi, T., Andreas Bærentzen, J., Sigmund, O.: 3d interactive topology optimization on hand-held devices. Structural and Multidisciplinary Optimization pp. 1-7 (2014)

8. Sigmund, O., Maute, K.: Topology optimization approaches. Structural and Multidisciplinary Optimization 48(6), 1031-1055 (2013)

9. Zhou, M., Rozvany, G.: On the validity of eso type methods in topology optimization. Structural and Multidisciplinary Optimization 21(1), 80-83 (2001) 